\title{
The Influence of Gender Matching and Result Feedback on University Students Cooperative Behavior
}

\author{
Yichen Pan \\ Chongqing Industry \& Trade Polytechnic \\ Chongqing, China 408100
}

\author{
Wei Wang* \\ Faculty of Teacher Education \\ Yangtze Normal University \\ Chongqing, China 408100 \\ *Corresponding Author
}

\begin{abstract}
The study focused on gender pairing and results feedback of the cooperative behavior of college students. Randomly selected 84 college students, Males and females equally divided, using 3 (male - male pairing, male - female pairing and female - female pairing) multiply by 2 (feedback, no feedback results) completely randomized design, the dependent variable indicators was cooperation behavior frequency. The results showed that: (1) the cooperative behavior of university students on gender pairing had a significant difference; the frequency of cooperative behavior was higher in the case of female. (2)The results feedback affect cooperative behavior frequency, results feedback of the average cooperative behavior frequency was higher than no results feedback. (3)The interaction of gender pairing factors and results feedback was not significant.
\end{abstract}

Keywords-college students; cooperative behavior; gender pairing; results feedback

\section{INTRODUCTION}

Cooperation is a common prosocial behavior in life. The so-called cooperation means that two or more people coordinate activities in order to achieve a common goal, so as to promote a behavior that is beneficial to both themselves and others. In the past decades of research, many studies have been conducted on the conditions or influencing factors of cooperation.

In researching the influencing factors of cooperative behavior, many researchers discussed such factors as the characteristics of the task, the characteristics of the group, and the characteristics of the individual. Rapoport (1995) discovered early on that in the face of real opponents, the frequency of cooperation of participants with feedback increases, and the frequency of cooperation of participants without feedback decreases [1]. Kelly and others found that partners will show competitive behavior when their partners first encounter competitors in real life, but when they meet partners again, they will show cooperation again [2]. A large number of studies have also found that communication and exchange can further influence cooperation, and foreign scholars also focus on the role of language exchange in research. Many researchers have found that children can achieve greater success in collaborative tasks if their peers can plan and negotiate in advance. Sally (1995) found in the study of social dilemmas that when face-to-face communication occurs, this situation can develop group identity and strengthen cooperation [3].

Matching methods also affect cooperation. Rogoff (1998) compared the effectiveness of collaboration between children and parents and their peers. The results show that children and adults tend to use complex strategies, and the effect of pairing adults and children is significantly better than that of children and children [4]. At the same time, children with complementary abilities can accomplish tasks more efficiently through mutual cooperation than children who are not complementary. Among them, the cooperation between children with weak ability and children with strong ability will also promote the improvement of the cognitive level of children with weak ability. However, for children with greater ability, the situation becomes more complicated. Sometimes they may benefit more from it, but sometimes they may also regress. The experiments of Siegler (2002) show that this is directly related to children's self-confidence, that is, the children with stronger self-confidence are more likely to show progress, and children with less selfconfidence are more likely to accept the reasoning of their peers even if they The level of reasoning is lower than its own level, thus showing a regression.

In the study of gender impact studies, Lindskold found that there were no gender differences in the fourth- and fifthgrade students' cooperative behavior in the Prisoner's Dilemma game. Madsen found that children aged 4 to 5 had no gender differences in the tug-of-war competition. In some studies that found gender differences, women were found to be more cooperative. The study by Knight and others found that there is a clear gender difference in children's perception of the value of competition and cooperation. The specific manifestation is that in the cooperation and competition, girls prefer to choose cooperation, boys prefer to choose to compete with each other, but later studies have shown that boys are more inclined to cooperate. [5] 
In recent years, the study of children's prosocial behavior has gradually entered a prosperous period. Cooperative behavior has also received increasing attention from psychologists. Domestic research on the factors affecting cooperative behavior has made more progress. For example, Chen Sijing (2015) began to study the influence of thirdparty punishment on cooperative behavior [6]. Ding Fang et al. (2016) studied the influence of emotional comprehension and emotional state on the cooperative behavior of primary school children [7]. Lu Yang et al. (2016) studied the influence of cooperation index and social distance on cooperative behavior [8]. Cui Liying et al. (2017) studied the influence of morality and relationship punishment on cooperation behavior of junior high school students in the public goods dilemma [9]. Wang Xiaofang et al. (2018) studied the influence of network structure heterogeneity on cooperative behavior [10].

However, there are still many problems in this area of research: (1) In the past, empirical research on cooperative behavior focused on children. The researchers gave children more attention. In the face of the current society's doubts about college students cannot be well adapted to social development, do not understand the new issues of cooperation, the study of cooperation behavior of college students as the research object is indispensable. (2) There are many areas of inconsistency in the results of the study. If the study of gender is based on whether men are more cooperative or women are more cooperative, there are still many opinions. There is still a lack of research on the influence of different genders on this social relationship on cooperative behavior. (3) In the past, many results showed that communication led to a significant increase in the cooperation rate, and the situation of forced communication was even more so. The feedback of the cooperation results is a kind of silent communication. Whether or not it has an impact on the cooperation behavior of the participants is also worthy of further attention.

Specifically, this study aims to verify the following hypotheses: (1) In the cooperative behavior of college students, there may be gender-promoting behaviors (ie, heterosexual gender-matching cooperative behavior is higher than same-sex matching); (2) Participants in the context of result feedback The frequency of cooperative behavior was higher than the frequency of participants' cooperative behavior under the condition of no-response feedback. (3) The effect of gender-matching factor on college students' cooperative behavior scores was influenced by the result feedback conditions, that is, the interaction between the two was significant.

\section{EXPERIMENTAL METHODS}

\section{A. Test}

From a university, 84 university students were randomly selected, 42 boys and 42 girls, with an average age of 22 years. Subjects' intelligence, hearing, and language were normal and there was no abnormal social behavior. Considering the influence of the relationship between the subjects on the cooperative behavior, the pre-inquiry ensures that the subjects do not recognize each other and that the subjects have no preference for the letter cord.

\section{B. Experimental Materials}

$6 \mathrm{~cm} \times 6 \mathrm{~cm}$ pieces of paper cards. The card is printed with the black letter $\mathrm{C}$ or $\mathrm{D}$. The letter $\mathrm{C}$ or $\mathrm{D}$ is printed in the middle of the card. This material is designed based on the materials used in the "Comrade Adolescent Cooperative Behavior Study", which is more common in the field of cooperative research in the past.

\section{Experimental Procedure}

1) Experimental design: This experiment uses 3 (malemale pairing, male-female pairing and female-female pairing) $\times 2$ (with result feedback, no result feedback) completely randomized design.

2) Experiment situation: A table was placed in the middle of the room. Only the main test and the test were taken. The main test was sitting on one side of the table. The two participants were sitting on both sides of the main test. The experiment simulates the "decision dilemma" gameplay. This game evolved from the "Prisoner's Dilemma" experiment conducted by Ruth and others in 1957. In the experiment, the subject had 12 cards in each hand, of which 6 cards were marked with the letter $c$ and 6 cards with the letter $\mathrm{d}$. Each time the game is played, both participants are asked to send a card to the main test. When the card was sent out, the participant could not see the letters on the other participant's card and could not speak.

3) Experimental procedure: The selected subjects were grouped and experimented under different guides.

Guideline 1: Now let's play a game for two of us. The rules of the game are as follows: Each player has 12 cards in his hand, of which 6 are marked with the letter $c$ and 6 are marked with the letter d. Each time the game is played, two players will each play a card (put the card upside down in my hand). If both are out of c, everyone will get 3 points. If one is out of $\mathrm{c}$, one is out of $\mathrm{d}$, one out of $\mathrm{c}$ gets 1 point, $\mathrm{d}$ out of 4 points. If both are out of $\mathrm{d}$, then both get 0 points. You can't see the letters on the other card, nor can you speak. After the game is over, the prize will be distributed to the first few experimenters based on each person's final score, hoping that everyone will strive for the final high score. The game is played in two rounds, 6 times per round, for a total of 12 times. At the end of each game, your choice will be announced.

Guideline 2: Now let's play a game for two people. The rules of the game are as follows: Each player has 12 cards in his hand, of which 6 are marked with the letter $c$ and 6 are marked with the letter d. Each time the game is played, two players will each play a card (put the card upside down in my hand). If both are out of c, everyone will get 3 points. If one is out of $\mathrm{c}$, one is out of $\mathrm{d}$, one out of $\mathrm{c}$ gets 1 point, $\mathrm{d}$ out of 4 points. If both are out of $\mathrm{d}$, then both get 0 points. You can't see the letters on the other card, nor can you speak. After the game is over, the prize will be distributed to the first few experimenters based on each person's final score, 
hoping that everyone will strive for the final high score. The game is played in two rounds, 6 times per round, for a total of 12 times. At the end of each game, the opponent's choice will not be announced.

After the preparation work is done, let the participants try several times to see if the participants really understand the rules of the game and then officially start the experiment. Give the top prizes after the experiment. Finally, the data were statistically analyzed.

4) Dependent variable and its measurement index: Operational definition of cooperative behavior: When the subject chooses $\mathrm{c}$, it is cooperative behavior.

Cooperative Behavior Score: The cumulative score of cselection for each participant in 12 games, one for each c. The higher the score, the more cooperative behavior. Finally, the results were statistically analyzed using SPSS19.0.

\section{RESULTS AND ANALYSIS}

The analysis of variance showed that the main effects of gender matching were significant, $F(2,36)=7.385$, $\mathrm{p}=0.002<0.05$; the main effects of the feedback factors were significant, $\mathrm{F}(1,36)=8.720, \mathrm{p}=0.006<0.05$ The interaction between gender matching and result feedback was not significant, $\mathrm{F}(2,36)=0.066, \mathrm{p}=0.936>0.05$. The average and standard deviation of cooperative behavior under gender matching are shown in "Table I". The average and standard deviation of the cooperative behavior under the feedback results are shown in "Table II". The average of the cooperative behavior scores under the feedback situation is significantly higher than the average of the cooperative behavior scores without the feedback of the results.

TABLE I. AVERAGE AND STANDARD DEVIATION OF COOPERATIVE BEHAVIOR IN GENDER MATCHING

\begin{tabular}{|l|l|l|}
\hline & M & SD \\
\hline Male-Male & 11.79 & 1.25 \\
\hline Male-Female & 13.5 & 1.22 \\
\hline Female-Female & 12.9 & 1.38 \\
\hline
\end{tabular}

TABLE II. AVERAGE AND STANDARD DEVIATION OF COOPERATIVE BEHAVIOR WITH RESULT FEEDBACK

\begin{tabular}{|l|l|l|}
\hline & M & SD \\
\hline Feedback & 13.29 & 1.23 \\
\hline No feedback & 12.19 & 1.47 \\
\hline
\end{tabular}

Because there are three levels of gender matching, further multiple comparisons are performed. The results show that the cooperation behavior in the male and female situations is significantly higher than that in the male-male pairing situation $(\mathrm{p}=0.002<0.01)$. It is also significantly higher than that of male-male pairing $(\mathrm{p}=0.043<0.05)$, but there is no significant difference between male-female pairing and female-female pairing $(\mathrm{p}=0.428>0.05)$.

\section{DISCUSSION}

\section{A. Influence of Gender Matching on College Students' Cooperative Behavior Score}

There are significant differences between college students in cooperative behavior scores. The specific performance is that the cooperation scores of male-female and female-female pairings are significantly higher than that of male-male pairs, and that of female-female pairings and male-female pairings. The difference in cooperation scores is not significant, which is basically consistent with the theoretical hypothesis1 of the experiment. However, in this experiment, we did not find that the effect of gendermatching factors on college students' cooperative behavior scores was influenced by the result feedback conditions. This was inconsistent with the theoretical hypothesis 3 of the experiment, that is, there was no significant interaction between the two.

There is a special kind of "gender promotion" in the social promotion phenomenon, that is, for mature people with sexual awareness, the opposite sex has a special behavior promotion function higher than the same sex. As for the mechanism of this promotion, social psychologist Zahalak passed a study in 1965 . He pointed out that the reason for social promotion lies in the existence of others and the intensification of personal internal motivation. Friedman further explained that the reason that the existence of another person leads to an increase in internal drive is because it enhances the individual's consciousness of being evaluated, thereby transforming the behavioral context into an evaluation situation with external incentives. Inspired by the evaluation consciousness, it will directly improve the selfobservation, self-evaluation and self-regulation level of the individual, so that the behavioral process is actually a selfexpression process led by a high degree of selfconsciousness. In the case of being evaluated, the motivation of the individual to expect a positive evaluation is stimulated so that the behavior develops in a direction consistent with social identity. The stronger the consciousness of being evaluated, the better the effect. For those who are sensitive to sexual maturity, in certain circumstances, the evaluation of the opposite sex is obviously more important than the assessment of same-sex, and therefore the motivation is greater. In this study, cooperative behavior is a positive behavioral response. The personal reaction will affect the evaluation of the other party and the corresponding response generated by the other party's evaluation will determine the score of both parties. Under male-female pairing, adolescent men and women, especially boys, are more concerned with the evaluation of the opposite sex than men and women, because at this moment they pay more attention to their own self-display in front of the opposite sex, hoping to give the opposite sex a best image. . This is an impression management process that has a guiding role in personal behavior. When playing a "decision-making dilemma" game, when the other party realizes that cooperation is beneficial to both parties and gets a positive evaluation from each other, they will naturally make more cooperative behavior choices. From this we can see that there is a certain truth in the society of "men and women matching and working hard". 
The subjects selected in this experiment have entered adolescence and their gender awareness has been very strong. Therefore, "gender promotion" plays a more significant role.

As for female-male vs. male-male pairing, high cooperative behavior can be explained in terms of stereotyped gender roles. Adolescent Cooperation - There are several reasons for the formation of competitive gender roles: First, family influence. Parents put different expectations on boys and girls, enabling their children to develop towards their parents' desired gender roles so that their children can incorporate these expectations into their stereotypes of gender roles. At the same time, parental attitudes and gender role stereotypes can subtly influence children's gender roles. The second is the influence of social role expectations. Expectations of social roles often create social pressure, so children have a clear understanding of gender identity that belongs to a certain gender group. Third, the mass media, while emphasizing women's attachment and cooperation, more publicizes men's leading role and competitive awareness. Therefore, in the face of the "decision making dilemma", male college students' competitive awareness tends to become the dominant tendencies and pay more attention to the difference between their own and each other's scores. They firmly believe that the other party also has the same ideas; in contrast, female college students hope to obtain a balanced score. . Everyone will not lose too many points, so choosing to cooperate is easier. Girls in the follow-up games have a stronger tendency to cooperate than boys. Based on the above reasons, in the case of male-male pairing, the two sides have negative expectations of each other, and think that the other party's goal is exclusive, and they will take action to gain their own advantages. However, under the female-female pairing, the two sides have more positive expectations of each other. They believe that the goals of each other are the same, so they take effective cooperation to promote the realization of common goals.

Differences in gender matching can be used to eliminate gender biases (or gender discrimination) in job hunting in society. It is generally believed that boys should be independent, risk-taking, enthusiastic, competitive, and have a strong sense of achievement. In many social work, people tend to choose men more. From the experimental results, as long as there is participation of women, there will be better cooperative behavior, because women have strong dependence, strong security needs, strong cooperation, the pursuit of interpersonal harmony, strong interpersonal skills, and interpersonal relationships. Sensitive and other characteristics, therefore, to have better cooperation behavior, women's participation is very necessary.

\section{B. Effect of Result Feedback on College Students Cooperative Behavior Score}

The result feedback plays an important role in college students' cooperative behavior scores. The results showed that the mean cooperative behavior scores of male-male, male-female, female-female paired were higher than that of no-result feedback, which was consistent with experimental theoretical hypothesis2.
Social dilemmas are a common problem in the real world. This dilemma has two characteristics: (1) For each strategy maker, if they adopt uncooperative strategies, they may obtain more benefits than adopting cooperation strategies. (2) If all counterparties adopt a cooperative strategy, the individual gains more than all individuals adopt a noncooperative strategy; when everyone adopts a noncooperative strategy, the benefit of each decision maker is far less than When everyone cooperates with each other. The "decision dilemma" is a dilemma. How to make individuals take cooperative actions to maximize group interests is the focus of such issues. In the "dilemma of decision-making," mutual trust has become a necessary psychological condition for cooperation, because each person's behavior restricts each other and is also restricted by the other. When the subject is in a decision-making dilemma, first of all he should think in transposition. He will ask himself: "If I were the other person, what choice would I make?" If he predicted that the other person would choose $\mathrm{c}$ (confidence), his chances of choosing $\mathrm{c}$ would also increase. Following the principle of fair exchange, his behavior is consistent with his expectations of others. In repetitive game decisions, unilateral cooperation does not continue, which is also related to the final individual score, and the result can only lead to competition. The accidental cooperation between the two did not reflect mutual trust. This may be just a coincidence, in the next game, one or both parties may use it, which leads to competition. In repeated interactions, information from the entire context is not complete, so information about each other becomes an effective alternative to experience. Especially when individuals have the right to choose to trust or distrust people they interact with, individuals can choose to cooperate on this basis. Posting the results of the selection to both parties after each match will serve to verify the unintended judgment of the other party's information. This trend of cooperation will continue after the cooperation has received positive feedback.

From the point of view of behaviorism, prosocial behavior can not only allow us to obtain returns from society, others, and ourselves, but also can avoid being punished by society, others, and ourselves. This will encourage you to form positive social values, such as "having good things to do at the end", "helping people to help themselves, and survival to coexist". Social exchange theory is one of the theories that explains the causes of prosocial behavior. It believes that the interaction between people is essentially a process of social interaction. Individuals try their best to gain the maximum benefit and pay as little as possible. That is, through social exchange theory, they weigh potential costs and benefits and decide how to help. The higher the perceived cost, the more difficult it is to help others. The more rewards you get, the more likely you are to help. People may feel very guilty of the consequences if they do not help people they could have helped. In most cases, prosocial behavior is caused by people's basic emotions and personal courage. In fact, pro-social behavior is beneficial to recipients and givers. People have many ways to feel satisfied and happy. Pro-social behavior is just one way to make both parties happy. This has an enlightening significance for our understanding of the influence of the 
"returning to piety" culture. Society should encourage, praise and promote this behavior in many ways.

\section{CONCLUSION}

College students' cooperation behavior scores show differences in gender matching. As long as there are girls, cooperative behavior is high, which means that in terms of cooperation, "gender promotion" is more accurately defined as "female support".

Result feedback factors have an impact on the frequency of college students' cooperative behavior. There is feedback from the results that the average cooperative behavior frequency is higher than no-result feedback.

\section{REFERENCES}

[1] Rapoport A, Chammah A. Prisoner's dilemma: A study in conflict and cooperation. Ann Arbor: University of Michigan Press, 1995.

[2] Stingle, Sandra F. Cook, Harold, Age and Sex Differences in the Cooperative and Noncooperative Behavior of Pairs of American Children[J]. Journal of Psychology, 1985,119(4): 335.

[3] Sally, D, (1995), Conversation and cooperation in social dilemmas: A metaanalysis of experiments from , 1958 to 1992 [J]. Rationality and Societ, 7, 58-92.

[4] Rogoff, B., Kimber, W. Cooperative Learning as an Academic Intervention for Students with Mildis -abolities[J]. Focus on Exceptional Children, 1998, 31(4):1-8.

[5] Su Hong, Li Yanhua, Ren Yongmei. A review of the research on the influencing factors of cooperative behavior[J].Journal of Kunming University of Science and Technology(Social Sciences Edition),2005,(3):76-79.

[6] Chen SJ, He Y, Ma J H. The Third Party Punishment's Influence on Cooperative Behavior: An Explanation Based on the Activation of Social Norm[J]. Chinese Journal of Psychology, 2015, 47(03): 389405.

[7] Ding Fang, Fan Limin, Zhang Lu. The Influence of Primary School Children's Emotion Understanding Ability and Emotional State on Their Cooperative Behavior[J]. Journal of Suzhou College of Education, 2016, 33(02): 84-89.

[8] Lu Yang, Zhang Lei, Xu Bibo. The Influence of Cooperation Index and Social Distance on Cooperative Behavior[J]. Psychological Science, 2016, 39(02): 435-440.

[9] Cui Liying, He Xing, Luo Junlong, Huang Xiaojiao, Cao Yujia, Chen Xiaomei. The Impact of Moral and Relational Punishment on Collaborative Behavior of Junior High School Students in the Dilemma of Public Goods[J]. Chinese Journal of Psychology, 2017, 49(10): 1322-1333.

[10] Wang Xiaofang, Ke Jianhong. The influence of network structure heterogeneity on cooperative behavior[J]. Journal of Wenzhou University(Natural Science),2018,39(01):56-62. 